\title{
Subject Index Vol. 95, 1996
}

\section{ABMT 164}

Accelerated phase 117

Acute monocytic leukemia 102

my eloid leukemia 122,129

myelomonocytic leukaemia 144 Adhesion 243

Adult ALL 188

- $\quad$ respiratory distress syndrome 49

AIDS 193

Anaemia, haemolytic, autoimmune 112 Anthracycline(s) 87, 144, 188 Apoptosis 13

Blast colony-forming cells 243 Bone marrow transplant 176

- $\quad$ - transplantation 157

Cardioplegia 63

Cardiotoxicity 87

Cat 213

c-Cbl 236

CD34 171,218

CD44 243

CD68 102

Cellular immunity 78

Central nervous system involvement 135

Cerebral malaria 78

Chelation 26

Chelators 70

Chemotherapy 70, 144, 188

Chromosomal abnormality 148

Chromosome 13218

Chromosomes 95

Chronic myelogenous leukemia 117

- $\quad$ neutrophilic leukemia 140

Classification 181

Complications 26

Cord blood 171 Cryopreservation 171 Cryptocrine signaling 263 Cytogenetics 181 Cytokine(s) $107,193,204,224$

Deferiprone 37, 78 Deferoxamine 37, 49, 66, 87 Density separation 171 Desferrioxamine 63

- $\quad$ B 78

Doxorubicin 87, 144

Enzyme-linked immunosorbent assay 112 Erythroid differentiation 229 Erythropoietin gene expression 248

FAB classification 102 FLT3 receptor 218 Free radicals 63, 87 
GATA transcription factor 248

GATA1 229

G-CSF 164

Gene therapy 213

GM-CSF 236

Graft-versus-host disease 157

Graft-versus-leukemia 157

Granulocytic sarcoma 129

Grb2/Ash 236

Growth factor 224

Hematopoiesis 204, 213, 218, 243, 257 Hem(at)opoietic progenitors 164, 171

- $\quad$ stemcell(s) 213,218

Hemopoietic growth factors 95

Hepatic iron stores 37

Homologous viral interference 199

Hydroxyl radical 49

Hydroxypyridinone(s) 6, 13

Hydroxyurea 117

Hypotriploidy 148

IL-1 107,268 IL-3 164 IL-4 107 IL-6 107,268 Immunodeficiency 204 Immunoglobulins, IgG, IgA 112 Interferon alfa-2b 117 Iron 66,70

chelation 6, 63, 78

chelators $13,49,78,87$

overload 13,26,37,87 Iron-chelating therapy 37

JAK2 224

Ki-1 anaplastic large-cell lymphoma 135 KIT receptor 218

Leptomeningeal seeding 135 Leukemia 257 Leukemoid reaction 140 Lipid peroxidation 78

Long-term culture 171 Lymphadenopathy 129 Lymphoma 193 Lysozyme 102

277

MAIDS 204 Malaria 70, 78 Malignant lymphoma 181 Megakaryocytic leukemia 122

Megakaryocytopoilesis 257 Molecular biology 181 Monoclonal gammopathy 140 Multiple myeloma 140,144 Myelodysplastic syndrome(s) 95, 144, 148 Myelofibrosis 122 Myeloid metaplasia 122 Myeloproliferative disorders 107

Neuroblastoma 66 Neuroendocrine self antigens 263 NonproducerHIV-1 199

Oral activity 6

Packaging cells 199

PBLs transduction 199

Peripheral blood precursor cell transplants

176 Phosphorylation 224 Plasmodium falciparum 70, 78 - vivax 78 Prognosis 95

Proinflammatory cytokines 268 Promoter 248 Proto-oncogenes 193

Rabbit 176

Reactive oxygen products 49 Reperfusion injury 63 Reporter gene 248 Retroviral vector 199

Ribonucleotide reductase 13

Sepsis 268

Signal transduction 218, 224 Simulation 213 Stemcell(s) 229,243 
- $\quad$ - factor $171,224,257$

Stochastic model 213

Suppression 204

Survival 26

T cell tolerance 263 Thalassemia 37 Thrombocythemia 107, 122 Thrombocytosis 107 Thymus epithelium 263 Toxicology 87 Transient transfection 248 Tumor suppressor genes 193 therapy 66 Tyrosine kinase 218

phosphorylation 236

Vav 236 Viruses 193

llq23 144

278

Subject Index Vol. 95, 1996 